\title{
Alianza estratégica Universidad y Colegios de Contadores Públicos del Perú para los procesos de certificación profesional
}

\author{
Mg. Elsa Esther Choy Zevallos
}

Docente Asociada de la Facultad de Ciencias Contables

\section{RESUMEN}

En los últimos años, en nuestro pais, el crecimiento de universidades con facultades o escuelas profesionales en contabilidad ha sido muy importante. Sin embargo, la falta de uniformidad en los planes de estudio de la carrera de contador público y la existencia de variados métodos para obtener los títulos profesionales en cada institución, son problemas que influyen en la calidad de la formación profesional.

El propósito de esta publicación es establecer la necesidad e importancia de la certificación para el desarrollo y mejora continua de la profesión contable; asimismo, la participación de las universidades en los procesos de acreditación de programas académicos y certificación de profesionales, especialmente en la carrera del contador público. Presenta un estudio descriptivo y de análisis, utilizando información e instrumentos legales relacionados con la certificación profesional, aplicable al contador público colegiado del Perú.

La evaluación por competencias es un proceso continuo que permite evidenciar la capacidad del ejercicio profesional, esto es posible si el profesional ha recibido una formación por competencias. Al final del presente trabajo, presentamos un perfil de alianza estratégica entre universidad y colegios profesionales; $y$, además, se adjunta un modelo de análisis funcional de la norma de competencia, desarrollado por el CCPL en un taller de capacitación sobre certificación profesional.

La universidad requiere de un cambio enmarcado dentro del campo de la mejora continua de la calidad de la enseñanza y de la calidad de la investigación, ambos constituyen la base para aspirar a la excelencia institucional. La certificación profesional es un proceso que permite a un profesional el reconocimiento de su desempeño y práctica profesional de acuerdo con los criterios de calidad, tales como: conocimientos, habilidades, actitudes y valores que posee para el buen desempeño de su práctica profesional. Existe una relación muy directa en cuanto a la participación de las universidades en los procesos de certificación profesional, teniendo en cuenta que la responsabilidad de éstas últimas está enmarcada en la formación del profesional por competencias.

Palabras clave: Certificación, recertificación, competencias profesionales y competencias laborales. 


\section{INTRODUCCIÓN}

La educación superior del siglo XXI pasa por una serie de desafíos y dificultades, producto del entorno cambiante, como son la globalización y la búsqueda de posicionamiento efectivo dentro de la sociedad del conocimiento.

La acreditación y certificación de la calidad educativa en el Perú tiene su génesis con la promulgación de la Ley General de Educación. Con la Ley No 28740, Sistema Nacional de Evaluación, Acreditación y Certificación de la Calidad Educativa (SINEA$\mathrm{CE}$ ), se inicia el camino a la acreditación de la calidad de las instituciones educativas y de sus programas; siendo las universidades y sus carreras profesionales y programas de posgrado competencia del Consejo Nacional de Evaluación, Acreditación, Certificación de la Calidad de la Educación Universitaria - CONEAU.

La acreditación es un proceso mediante el cual las instituciones educativas y sus programas son reconocidos por una instancia externa, esta instancia es el CONEAU; su finalidad es asegurar que dichas instituciones y programas cumplan con normas apropiadas de calidad e integridad. Es un proceso colegiado basado en la autoevaluación y la evaluación por pares, para mejorar la calidad académica y la aceptación social.

Por su parte, la certificación profesional es considerada como un proceso que se realiza para verificar que un individuo está calificado para practicar una profesión. Entre sus objetivos destacan: asegurar que los miembros de una profesión estén actualizados y calificados para practicar cierto campo profesional; mantener las competencias adecuadas para satisfacer las expectativas del contratante; e incrementar el estatus ante los directivos de las instituciones que los contra- tan, así como de la sociedad en general. En tal sentido, la certificación verifica el conocimiento y habilidades de los individuos y la acreditación valida la institución educativa donde los adquirió.

Actualmente, en nuestro país existe gran preocupación acerca de estos temas; precisamente, este trabajo de investigación centra su atención en describir los procesos de certificación profesional para el contador público colegiado y la importante participación de la universidad en la formación del profesional por competencias.

\section{ANTECEDENTES LEGALES}

La Ley 28044, Ley General de Educación, del 29-07-03, crea el Sistema Nacional de Evaluación, Acreditación y Certificación de la Calidad Educativa -SINEACE.

La Ley 28740, Ley del Sistema Nacional de Evaluación, Acreditación y Certificación de la Calidad Educativa -SINEACE del 2305-06, señala:

\footnotetext{
Art. 11. "La certificación es un proceso público y temporal de las competencias adquiridas dentro o fuera de las instituciones educativas para ejercer funciones profesionales y laborales, que es otorgada por el Colegio Profesional correspondiente, a solicitud del interesado".
}

Ley N. ${ }^{\circ} 28951$, de Actualización de la Ley N.o 13253, Ley de Profesionalización del Contador Público y Creación de los CCPP, señala en sus artículos:

Art. 60. Atribuciones de los Colegios de Contadores Públicos.

c) Organizar y llevar el registro de los miembros de la Orden colegiados, por especialidades, para efectos de la certificación y recertificación en la 
forma establecida por el estatuto y el reglamento interno.

Art. 13º. Certificación y Recertificación.

La Certificación y Recertificación de los Contadores Públicos Colegiados a nivel nacional, está a cargo de la Junta de Decanos de Colegios de Contadores Públicos del Perú, en su calidad de máximo organismo representativo de la profesión contable dentro del país y en el exterior, conforme a lo dispuesto por el Decreto Ley No 25892 y su reglamento aprobado por Decreto Supremo No 008-93-JUS.

DecretoSupremo N. ${ }^{\circ} 018-2007-E D(10.07 .07)$, Aprueba el Reglamento de la Ley N. ${ }^{\circ} 28740$ (Ley del SINEACE).

Artículo $21^{\circ}$. Dispone que las entidades certificadoras de competencias profesionales, entre otros requisitos, deben disponer de un conjunto de instrumentos actualizados que permitan la evaluación y certificación de competencias laborales y/o profesionales del perfil laboral seleccionado, o de la profesión respectiva, elaborados de acuerdo con los Análisis Funcionales preparados según parámetros nacionales e internacionales de evaluación y certificación de competencias.

\section{LA CALIDAD EN LA EDUCACIÓN SUPERIOR UNIVERSITARIA}

Se entiende por calidad al conjunto de características inherentes a un producto o servicio que cumple los requisitos para satisfacer las necesidades preestablecidas. De este modo, una carrera universitaria de calidad define claramente su misión y visión en función de sus grupos de interés, estos propósitos abarcan las actividades confiadas por la sociedad.

Así, tenemos que la misión y visión de una de las universidades más importantes del Perú, son:

\section{Misión}

Somos una universidad dedicada a la generación y transmisión del conocimiento y a la formación de profesionales emprendedores y altamente competitivos, capacitados para ser exitosos en la sociedad global y dotados de sólidos valores éticos y humanísticos, promoviendo la investigación aplicada a la solución de los problemas sociales y contribuyendo al desarrollo del país.

\section{Visión}

Somos una universidad con alto nivel en la creación y desarrollo de conocimientos para la formación de competencias con ágil capacidad de innovación y respuesta a los agentes socioeconómicos del entorno, con efectividad en la transferencia y generación de innovación de los procesos académicos y con un fuerte y sostenible vínculo de colaboración con las unidades del sector socioeconómico con un alto impacto social.

La Declaración Mundial sobre la Educación Superior en el siglo XXI amplía esta definición en cuanto a su evaluación, involucrando más categorías de análisis:

"La calidad de la enseñanza superior es un concepto pluridimensional que debería comprender todas sus funciones y actividades: enseñanza y programas académicos, investigación y becas, personal, estudiantes, edificios, instalaciones, equipamiento y servicios a la comunidad y al mundo universitario. Una autoevaluación interna y un examen externo realizados con transparencia por expertos independientes, en lo posible especializados en lo internacional, son esenciales para la mejora de la calidad. Deberían crearse instancias nacionales independientes, $y$ definirse normas comparativas de calidad, reconocidas en el plano internacional. Con miras a tener en cuenta la diversidad y evitar la uniformidad, debería prestarse la atención debida a las 
particularidades de los contextos institucional, nacional y regional. Los protagonistas deben ser parte integrante del proceso de evaluación institucional".

"La calidad requiere también que la enseñanza superior esté caracterizada por su dimensión internacional: el intercambio de conocimientos, la creación de sistemas interactivos, la movilidad de profesores y estudiantes y los proyectos de investigación internacionales, aun cuando se tengan debidamente en cuenta los valores culturales y las situaciones nacionales".

"Para lograr y mantener la calidad nacional, regional o internacional, ciertos elementos son especialmente importantes, principalmente la selección esmerada del personal y su perfeccionamiento constante, en particular mediante la promoción de planes de estudios adecuados para el perfeccionamiento del personal universitario, incluida la metodología del proceso pedagógico, y mediante la movilidad entre los países y los establecimientos de enseñanza superior y entre los establecimientos de educación superior y el mundo del trabajo, así como la movilidad de los estudiantes en cada país y entre los distintos países. Las nuevas tecnologías de la información constituyen un instrumento importante en este proceso debido a su impacto en la adquisición de conocimientos teóricos y prácticos”.

Según la UNESCO: "La calidad es la adecuación del Ser y Quehacer de la Educación Superior a su Deber ser". Es decir, la Misión (Deber Ser), al igual que los planes y proyectos que de ella se deriven, son evaluados en cuanto a su pertinencia; el funcionamiento (Qué hacer) es evaluado en términos de eficiencia; y los logros y resultados (Ser) son evaluados en cuanto a su eficacia.

\section{DISEÑO CURRICULAR Y PROCESOS DE ENSEÑANZA}

El proceso académico debe establecer la efectividad de su puesta en práctica, es decir, si la institución hace lo que promete, si lo que hace lo hace bien y si provee a sus alumnos de los elementos formativos que les permitan lograr las competencias académico-profesionales de salida postuladas; todo lo cual debiera ser demostrable.

En esa perspectiva, cabe examinar la intencionalidad del currículum, la pertinencia de sus contenidos y la congruencia de su estrategia formativa con el perfil profesional de salida (conocimientos, competencias y actitudes a lograr). Para lo cual debemos tener en cuenta las siguientes interrogantes:

- ¿Toma en cuenta el perfil académico real de los alumnos con los que tiene que aplicarlo?

- ¿Considera los requerimientos del mercado de trabajo?

- ¿Su enfoque integra los aspectos de formación profesional y los de desarrollo personal?

- ¿Propicia en el alumno la creatividad, la investigación, la capacidad de crear fuentes de trabajo y la responsabilidad social?

- ¿Dedica la entidad y su cuerpo docente una adecuada atención a la preparación de los planes de estudio?

- ¿Cuál es la participación de los docentes en la formulación y evaluación del currículo?

- ¿Existe algún tipo de evaluación del perfil profesional que permita demostrar las competencias adquiridas del egresado?

Al examinar el currículo se debe tener en cuenta:

- La coherencia entre la propuesta académica y lo exigido para ser admitido como alumno del programa. 
- La coherencia de sus objetivos y estrategias de aprendizaje con los medios y recursos utilizados para plasmarlos.

- Mecanismos y prácticas que la institución pone en juego para estimular o exigir a que sus alumnos cumplan con sus responsabilidades académicas.

- Modalidades y plazos en que se traduce la adecuación programática a la menor disponibilidad de tiempo de los alumnos que trabajan.

- La eficacia del control de los aprendizajes de los alumnos, la pertinencia y validez de los criterios y forma de evaluarlos y de verificar si logran las competencias profesionales establecidas en el perfil del egresado.

Este último punto, podría comprobarse en el momento de la evaluación del profesional que solicita su certificación profesional.

\section{TRATAMIENTO CURRICULAR DE LAS COMPETENCIAS BÁSICAS Y PROFESIONALES}

El tratamiento curricular de las competencias debiera aplicarse de forma obligatoria en la educación superior, las universidades deben utilizar un modelo curricular organizado en contenidos transversales, actualmente es esta la tendencia en algunas instituciones, de manera que integren los esfuerzos formativos y promuevan el desarrollo de los diferentes niveles de dominio de las competencias a lo largo de la formación profesional del estudiante. Los sílabos deben ser diseñados por competencias y emplear la evaluación continua.

El tratamiento de las competencias y capacidades en los niños, niñas y adolescentes (educación básica) es incipiente en las escue- las públicas, mientras que en las privadas los alumnos se incorporen cada vez más a esta cultura del aprendizaje por competencias, al igual que sus profesores.

\section{COMPETENCIA PROFESIONAL}

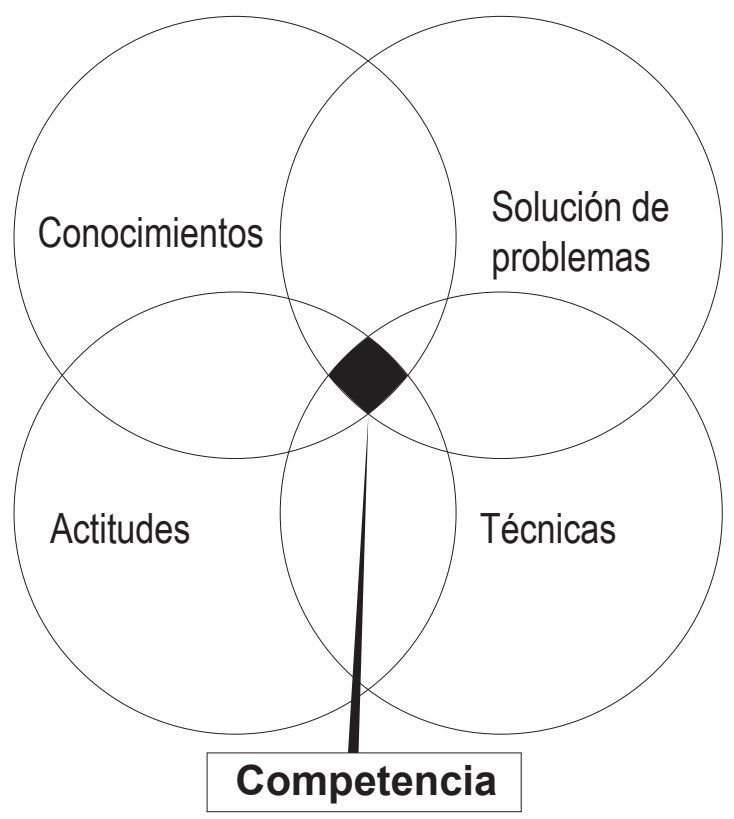

\section{UNA NORMA DE EDUCACIÓN PROFESIONAL CONTINUA}

Los colegios de profesionales son entidades de derecho público, autónomos, y se conforman por individuos que persiguen un propósito común para su gremio. El sentido de pertenencia a un grupo es uno de los grandes ideales del ser humano. Asimismo, la búsqueda del aprendizaje es un atributo que distingue a estas agrupaciones profesionales, persiguiendo que sus agremiados sean aptos para ofrecer, en su actividad, servicios que sean útiles para la sociedad.

En una sociedad que tiene tan arraigado el sentido material, el patrimonio de los individuos es uno de sus mayores requerimientos para conservar su tranquilidad y para el desarrollo de su existencia, es en esto en lo 
que radica la verdadera esencia de estas profesiones y así como el médico que tiene a su cuidado la vida de los seres, y que requiere estar de manera permanente actualizando sus conocimientos, los profesionales contables encargados de cuidar el patrimonio de los individuos requieren de constante actualización.

En el Perú debería ser obligatorio cumplir con una norma de educación profesional continua para los contadores públicos afiliados a los colegios profesionales

La existencia de normas y principios establecidos por la sociedad obligan al profesional a conocerlas, aplicarlas y cumplirlas. El ámbito profesional es cada vez más complejo debido a la gran cantidad de información que se requiere procesar, proveniente del ámbito nacional o internacional, por lo tanto, se vuelve una exigencia la capacitación del profesional para entenderla, analizarla y aplicarla, ello evidencia la obligación de cumplir con los mínimos requerimientos de aprendizaje que eviten riesgos en el cuidado patrimonial.

\section{LA CERTIFICACIÓN PROFESIONAL DEL CONTADOR PÚBLICO}

\section{Certificación Profesional}

La certificación es el acto mediante el cual una persona consta que posee los conocimientos, habilidades, destrezas y valores acordes con las necesidades del mercado laboral.

En nuestro país, la certificación profesional del contador público está a cargo de la Junta de Decanos de los Colegios de Contadores Públicos del Perú -JDCCPP, con este proceso se avala la pericia de los profesionales de esta carrera, con base en la preparación, capacitación, evaluaciones periódicas y actualización profesional permanente.
Consiste en un nuevo nivel profesional, ya que es la constancia que avala los conocimientos, habilidades y destrezas requeridos para el ejercicio de la profesión dentro del sector empresarial, además de ser una garantía de calidad en cuanto a la capacidad técnica y a la conducta ética del profesional contable que la obtiene.

\section{Certificación Profesional Extraordinaria}

Fue un beneficio para la profesión contable en nuestro país, en virtud de la CLÁUSULA DEL ABUELO, es decir, para aquellos contadores públicos con tres o más años de colegiatura y experiencia profesional, se les otorgó la certificación profesional, bajo la modalidad de "Portafolio Personal, en un tiempo limitado, en todo el ámbito nacional.

Actualmente, está vigente la certificación profesional ordinaria, se espera una evaluación por especialidades, así por ejemplo podría considerarse las siguientes:

\section{Especialidades a certificar}

- Contabilidad Empresa Privada

- Contabilidad Gubernamental

- Auditoría Financiera

- Auditoría Gubernamental

- Auditoría Integral

- Finanzas

- Tributos

- Costos y Presupuestos

- Investigación Aplicada

La certificación tiene vigencia cuatro años, al término de los cuales el profesional deberá recertificarse. Sería importante la participación compartida de las universidades y los colegios profesionales, en la etapa de certificación profesional, por tratarse de una evaluación formativa de las competencias, teniendo en cuenta que estas son adquiridas mayormente en estas instituciones educativas. 
A nivel nacional, se cuenta con 25 colegios departamentales y con una membresía cercana a los 50000 contadores, de los cuales, aproximadamente, 4000 están actualmente certificados.

\section{RECERTIFICACIÓN PROFESIONAL}

Es un proceso establecido para determinar si el contador público mantiene su competencia o la calidad de desempeño en la práctica a un estándar suficientemente elevado como para poder mantener su certificación.

En nuestro país, el proceso de recertificación se aplicará dentro de tres años aproximadamente, teniendo en cuenta que ha transcurrido cerca de un año de aquel primer profesional certificado. La Junta de Decanos tendrá a cargo dicho proceso, para lo cual deberá tener en cuenta ciertos criterios de calificación, que aún no están establecidos; sin embargo, para tener un alcance de cuáles pueden ser estos criterios, presentamos a continuación un ejemplo:

\section{Criterios de calificación}

- El mecanismo de recertificación se basa en la acumulación de unidades de evaluación, para este caso denominados créditos. Un crédito equivale a $(\mathrm{x})$ horas teóricas o $(\mathrm{x})$ horas prácticas de aquellos cursos que sean reconocidos como válidos para la recertificación.

- Los créditos acumulados se tomarán en cuenta a partir de los ( $\mathrm{x}$ ) ańos previos a la fecha de la solicitud de la credencial de recertificación y se exigirá como mínimo un total de $(\mathrm{x})$ créditos.

- El SINEASE, en coordinación con todas las instancias relacionadas, publicará oportunamente el calendario de eventos y actividades científicas conducentes a la obtención de créditos válidos para la certificación. En este rol deberá detallarse los contenidos principales y el número de créditos que otorga cada evento.

- Para la calificación del contador público colegiado se tomará en cuenta para la recertificación los siguientes rubros:

1. Participación y aprobación en los diferentes cursos válidos para la recertificación.

2. El criterio fundamental de la evaluación será la comprobación que el contador público colegiado se encuentre en un proceso de educación profesional continua.

\section{RESULTADOS FINALES}

\section{Alianza estratégica}

Cooperación a corto, y al menos en intención, mediano y largo plazos, que planifican, organizan, gestionan y controlan, dos o más sistemas organizacionales, en correspondencia con sus respectivas estrategias de desarrollo.

\section{PERFIL BÁSICO DE ALIANZA UNIVERSIDAD Y COLEGIO PROFESIONAL}

En la primera evaluación para la CERTIFICACIÓN, la relación existente entre universidad y colegio profesional es importante, toda vez que la formación del profesional respecto a las competencias base las recibe en la universidad. En este trabajo de investigación se sugiere una alianza estratégica entre universidad y colegios profesionales, donde el compromiso de la universidad sería una formación del profesional egresado en base a competencias. 


\section{CERTIFICACIÓN Y RECERTIFICACIÓN}

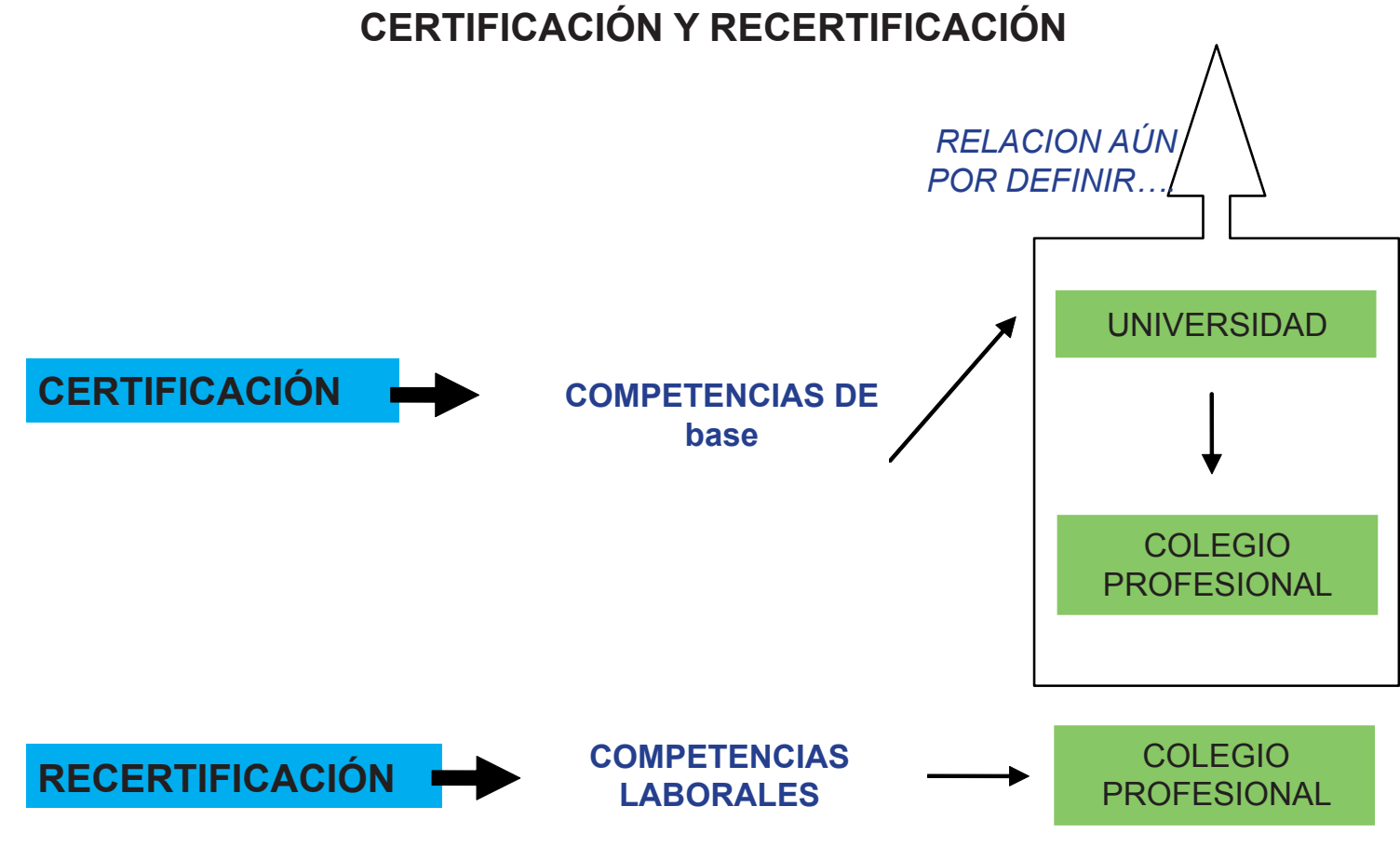

\section{COMPETENCIA PROFESIONAL}

Según M.T. Kane (1992), "Representa el grado al que una persona puede utilizar sus conocimientos, aptitudes, actitudes y buen juicio asociados a la profesión, para poder desempeñarse de manera eficaz en todos las situaciones que corresponden al campo de su práctica profesional".

El Colegio de Contadores Públicos de Lima realizó un taller a efecto de determinar cuáles serían las competencias a evaluar; en tal sentido, este evento académico desarrollo una norma de competencia a través de un análisis funcional.

\section{ANÁLISIS FUNCIONAL}

El análisis funcional es una de las formas de aproximación al contenido de las ocupaciones desde la óptica de las competencias. Permite reconocer, de manera general, que una persona es competente para hacer "algo" cuando demuestra que lo sabe hacer. Si ese algo tiene que ver con el trabajo, puede decirse que la persona es competente; es decir, la persona posee competencia laboral.

La competencia laboral es, entonces, uno más de los diferentes atributos que posee la persona y, por lo tanto, dicha competencia es identificable en la persona misma. 
MAPA FUNCIONAL

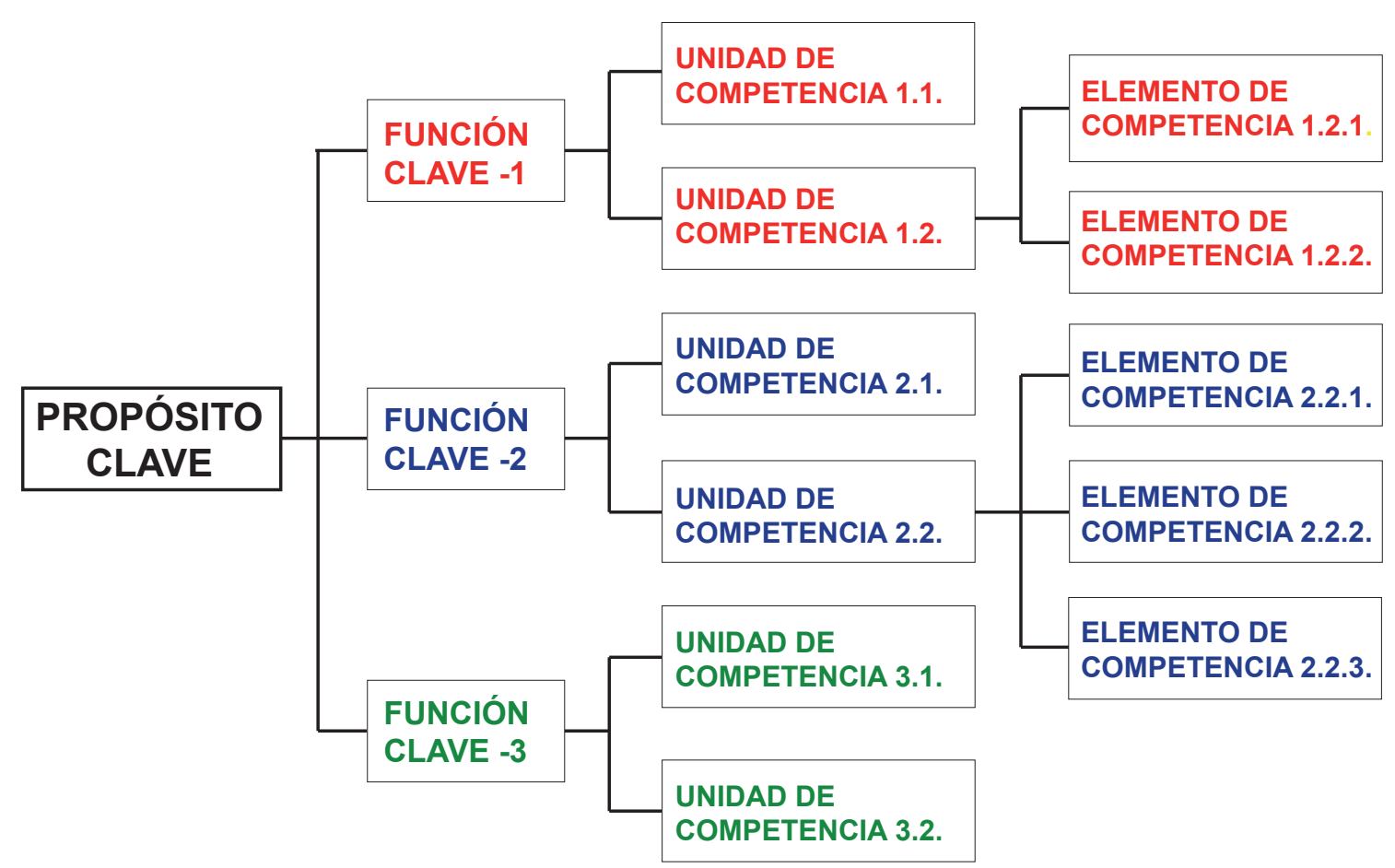

Caso práctico de evaluación de competencias a través del análisis funcional:

\section{PROPÓSITO DEL CONTADOR}

\section{PÚBLICO}

"Brindar servicio contable general e información de la situación económica y financiera de una entidad, en forma oportuna y confiable, de acuerdo a principios contables y normatividad vigente, con responsabilidad social en un entorno cambiante".

\section{FUNCIÓN 2}

Auditar Estados Financieros de acuerdo a normatividad vigente.

\section{UNIDAD 2.2}

Evaluar el sistema de control interno de acuerdo al giro de la entidad y a normas de auditoría

\section{ELEMENTO 2.2.3}

Elaborar la carta de control interno que incluya las observaciones y recomendaciones para la entidad.

La evaluación del elemento de competencia 2.2.3 requerirá la preparación de la respectiva NORMA DE COMPETENCIA. 


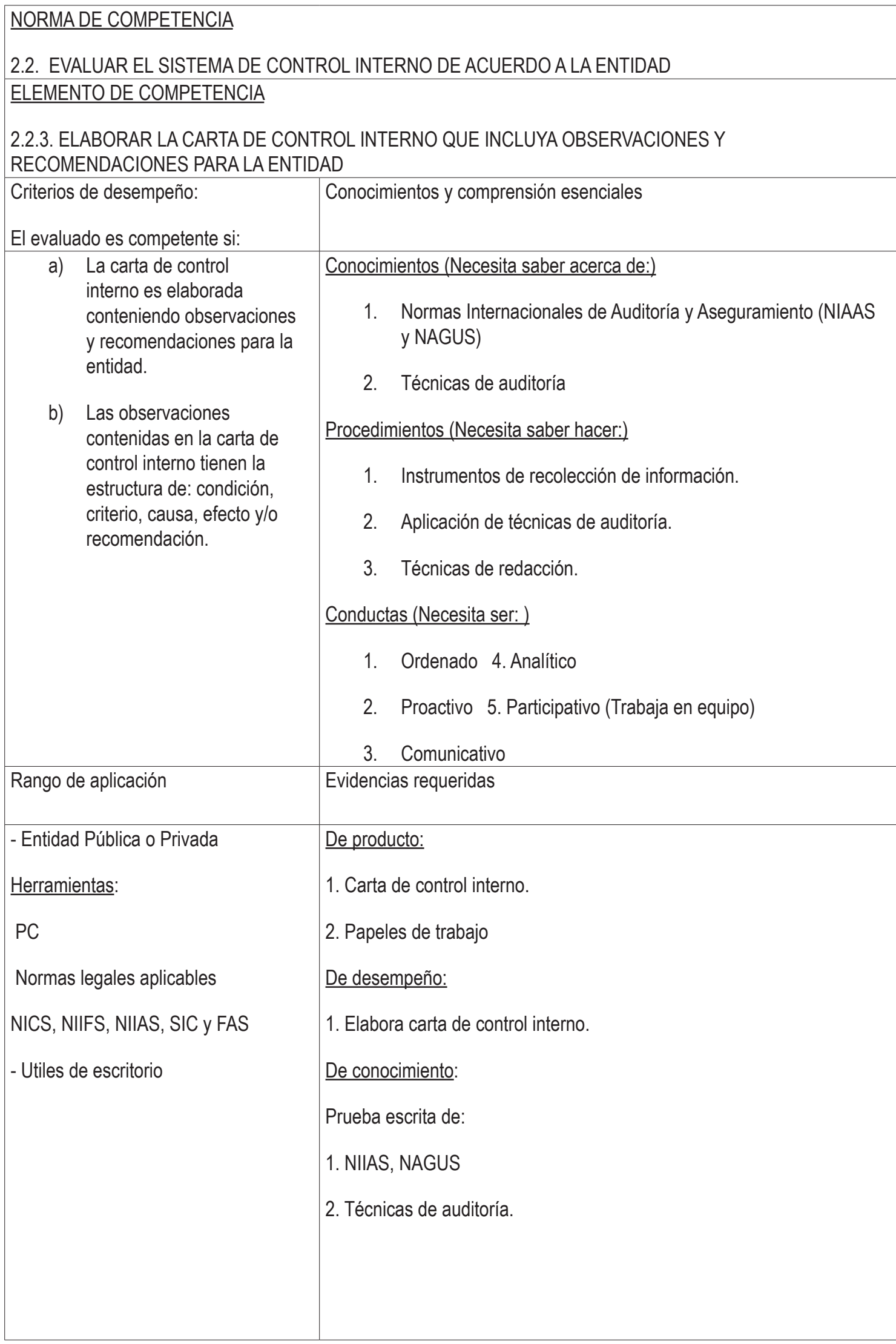

\section{QVIPURAMAYOC}




\section{CONCLUSIONES}

- El siglo XXI, considerado la era del conocimiento, evidencia un claro desfase de la universidad con la época, un cambio enmarcada dentro del campo de la mejora continua de la calidad de la enseñanza y de la calidad de la investigación, ambos constituyen la base para aspirar a la excelencia institucional.

- La educación profesional continua, la experiencia progresiva y la certificación profesional, son parte de la nueva cultura que redundará en el aumento de la calidad del ejercicio profesional.

- La certificación profesional es un proceso por el cual se otorga reconocimiento a un profesional por haber cumplido con los criterios de calidad, en relación a los conocimientos, habilidades, actitudes y valores que posee para el buen desempeño de su práctica profesional. Tiene un plazo de cuatro años, al término del cual el profesional deberá recertificarse.

- La recertificación profesional es después de transcurrido cuatro ańos de haber obtenido la certificación profesional, consiste en sustentar si el profesional mantiene su competencia o la calidad de desempeño profesional y laboral.

- La certificación y recertificación de los contadores públicos colegiados a nivel nacional, está a cargo de la Junta de Decanos de Colegios de Contadores Públicos del Perú, en su calidad de máximo organismo representativo de la profesión contable dentro del país y en el exterior.

- Los colegios profesionales comparten con el estado y las universidades, la responsabilidad de formar profesionales de calidad, conforme a la confianza que la sociedad ha depositado en ellos.

\section{REFERENCIAS}

García Rocha, J.A. (2005). Glosario de términos básicos en regulación y acreditación en educación superior virtual y transfronteriza.

González, L. Ayarza, H. Calidad, evaluación institucional y acreditación en la educación superior en la región latinoamericana y del Caribe. CINDA. Santiago de Chile.

\section{Informe MUNDIAL DE LA UNESCO}

Hacia la sociedad del conocimiento.

\section{Fuentes electrónicas}

www.ehu.es/cuadernosdegestion/documentos Gestión del conocimiento científicotécnico en la universidad.

www.madrimasd.org/informacionidi/bibliotecal

Gestión del conocimiento en universidades y organismos públicos. 